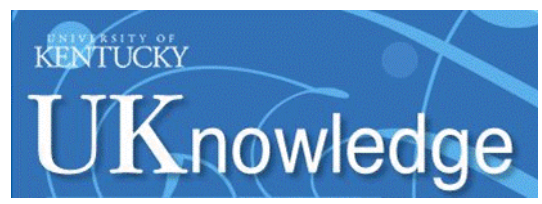

University of Kentucky

UKnowledge

$12-2015$

\title{
Crystal Structure of $(E)$-13-(pyrimidin-5-yl)parthenolide
}

Shobanbabu Bommagani

University of Arkansas for Medical Sciences

Narsimha Reddy Penthala

University of Arkansas for Medical Sciences

Sean Parkin

University of Kentucky, s.parkin@uky.edu

Peter A. Crooks

University of Arkansas for Medical Sciences

Follow this and additional works at: https://uknowledge.uky.edu/chemistry_facpub

Part of the Chemistry Commons

Right click to open a feedback form in a new tab to let us know how this document benefits you.

\section{Repository Citation}

Bommagani, Shobanbabu; Penthala, Narsimha Reddy; Parkin, Sean; and Crooks, Peter A., "Crystal Structure of (E)-13-(pyrimidin-5-yl)parthenolide" (2015). Chemistry Faculty Publications. 63.

https://uknowledge.uky.edu/chemistry_facpub/63

This Article is brought to you for free and open access by the Chemistry at UKnowledge. It has been accepted for inclusion in Chemistry Faculty Publications by an authorized administrator of UKnowledge. For more information, please contact UKnowledge@lsv.uky.edu. 


\section{Crystal Structure of (E)-13-(pyrimidin-5-yl)parthenolide}

\section{Digital Object Identifier (DOI)}

https://doi.org/10.1107/S2056989015021507

\section{Notes/Citation Information}

Published by Acta Crystallographica Section E: Crystallographic Communications, v. 71, part 12, p. 1536-1538.

This is an open-access article distributed under the terms of the Creative Commons Attribution (CC-BY) Licence, which permits unrestricted use, distribution, and reproduction in any medium, provided the original authors and source are cited. 


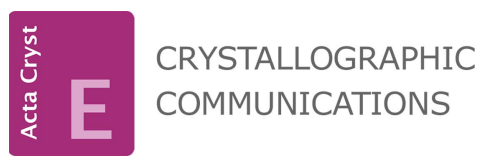

ISSN 2056-9890

Received 2 October 2015

Accepted 12 November 2015

Edited by G. Smith, Queensland University of Technology, Australia

Keywords: crystal structure; parthenolide; pyrimidine; Heck product

CCDC reference: 1436825

Supporting information: this article has supporting information at journals.iucr.org/e

\section{Crystal structure of $(E)-13-($ pyrimidin-5-yl)- parthenolide}

\author{
Shobanbabu Bommagani, ${ }^{a}$ Narsimha R. Penthala, ${ }^{a}$ Sean Parkin ${ }^{\mathrm{b}}$ and Peter A. \\ Crooks $^{\mathrm{a} *}$ \\ a Dept. of Pharm. Sciences, College of Pharmacy, University of Arkansas for Medical Sciences, Little Rock, AR 72205, \\ USA, and ${ }^{\mathbf{b}}$ Dept. of Chemistry, University of Kentucky, Lexington KY 40506, USA. *Correspondence e-mail: \\ pacrooks@uams.edu
}

The title compound, $\mathrm{C}_{19} \mathrm{H}_{22} \mathrm{~N}_{2} \mathrm{O}_{3}$, \{systematic name $(1 \mathrm{a} R, 4 E, 7 \mathrm{a} S, 8 E, 10 \mathrm{a} S$,$10 \mathrm{~b} R$ )-1a,5-dimethyl-8-[(pyrimidin-5-yl)methylidene]-2,3,6,7,7a,8,10a,10b-octahydrooxireno[ $\left[2^{\prime}, 3^{\prime}: 9,10\right]$ cyclodeca[1,2-b]furan-9(1aH)-one $\}$ was obtained from the reaction of parthenolide [systematic name $(1 \mathrm{a} R, 7 \mathrm{a} S, 10 \mathrm{a} S, 10 \mathrm{~b} R, E)-1 \mathrm{a}, 5-$ dimethyl-8-methylene-2,3,6,7,7a,8,10a,10b-octahydrooxireno[2',3':9,10]cyclodeca[1,2-b]furan-9(1aH)-one] with 5-bromopyrimidine under Heck reaction conditions, and was identified as an $E$ isomer. The molecule possesses ten-, five(lactone) and three-membered (epoxide) rings with a pyrimidine group as a substituent. The ten-membered ring displays an approximate chair-chair conformation, while the lactone ring shows a flattened envelope-type conformation. The dihedral angle between the pyrimidine moiety and the lactone ring system is $29.43(7)^{\circ}$.

\section{Chemical context}

Parthenolide (PTL) is a sesquiterpene lactone known to significantly target cancer stem cells, which are the putative roots of all types of cancer (Gopal et al., 2007). PTL has been isolated from several different plant species, feverfew leaf (Tanacetum parthenium) being one of the major sources (Awang, 1989). PTL exhibits a wide range of biological activities, such as anti-inflammatory, anti-bacterial, antifungal, and cytotoxic properties (Picman, 1986). Consequently, PTL was discovered to be capable of inducing robust apoptosis in primary acute myelogenous leukemia (AML) cells (Guzman et al., 2007), proving to be equally effective among all subpopulations within primary AML specimens, including leukemia stem cells (LSCs). Gopal et al. (2007) reported that PTL specifically depletes HDAC1 protein without affecting other class I/II HDACs (histone deacetylases). Nasim et al. (2008) reported the anti-leukemic activity of aminoparthenolide analogues. Han et al. (2009) reported on bioactive derivatives of Heck products of PTL. Recently, Penthala et al. (2014a) reported the anti-cancer activity of PTL-Heck products. Recently we (Penthala et al., 2014b) reported the crystal structure of $13-\{4-[Z-2-c y a n o-2-(3,4,5-$ trimethoxyphenyl)ethenyl]phenyl parthenolide, an analog of PTL, which was found to have the $E$ configuration at C-13. The interesting biological properties of PTL directed our attention to design and synthesize additional bioactive derivatives. In order to obtain detailed information on the structural conformation of the current molecule, including assignment of the absolute configuration of the four stereocentres, and to establish the geometry of the exocyclic double 
bond, a single crystal X-ray structure determination has been carried out.<smiles>C/C1=C\CC[C@@]2(C)O[C@H]([C@@H]3/C(=C\c4cncnc4)C(=O)O[C@@H]32)[C@@H]1O</smiles>

\section{Structural commentary}

The title compound is shown in Fig. 1. The PTL substructure of the molecule contains a ten-membered carbocyclic ring (chair-chair conformation) fused to a lactone ring (flattened envelope-type conformation), and an epoxide ring, as previously reported (Castañeda-Acosta \& Fisher, 1993). The title compound contains an E-exocyclic olefinic bond $\mathrm{C} 11=\mathrm{C} 13$. The pyrimidine ring is twisted out of the plane of the furan ring, making a dihedral angle of $29.43(7)^{\circ}$. The $\mathrm{C} 11=\mathrm{C} 13-\mathrm{C} 16$ bond angle of $127.89(16)^{\circ}$ deviates from the ideal value of $120^{\circ}$, but other bond lengths and angles are largely unremarkable. The four chiral carbon atoms in PTL were determined using 1354 quotients (Parsons et al., 2013) as follows: $\mathrm{C} 4(R), \mathrm{C} 5(R), \mathrm{C} 6(S), \mathrm{C} 7(S)$ for the arbitrary atomnumbering scheme used, and is consistent with previous studies (Penthala et al., 2013).

\section{Supramolecular features}

There are no classical hydrogen bonds and no $\pi-\pi$ interactions. There are a few $\mathrm{C}-\mathrm{H} \cdots \mathrm{N}$ and $\mathrm{C}-\mathrm{H} \cdots \mathrm{O}$ short contacts, but none that have the right geometry to be considered as non-classical hydrogen bonds. Intermolecular contacts thus appear to be limited to van der Waals interactions.

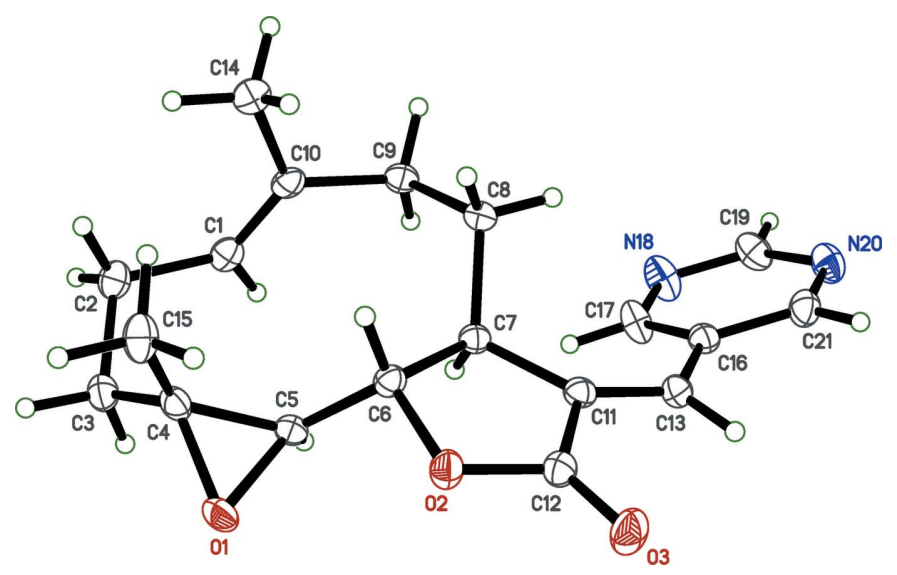

Figure 1

The molecular structure of the title compound with probability ellipsoids drawn at the $50 \%$ probability level.

\section{Database survey}

A search of the November 2014 release of the Cambridge Structure Database (Groom \& Allen, 2014) for the PTL substructure gave 24 hits. Two of these (PARTEN: Quick \& Rogers, 1976; PARTEN01: Bartsch et al., 1983) give the structure of PTL itself, with the remaining 22 being substituted variants of PTL. Of these substituted parthenolides, only four CSD entries: HORZOF (Penthala et al., 2014b), HUKLAB, HUKLEF (Han et al., 2009) and QILGEZ (Penthala et al., 2013), are substituted at the exocyclic double bond.

\section{Synthesis and crystallization}

Synthetic procedures: The title compound, containing the PTL substructure, was synthesized by the previously reported literature procedure (Han et al., 2009). In brief, parthenolide (1 mmol), 5-bromopyrimidine $(1.1 \mathrm{mmol})$, triethylamine ( $3.0 \mathrm{mmol})$ and $5 \mathrm{~mol} \%$ of palladium acetate were charged into dimethylformamide $(2 \mathrm{ml})$ at room temperature. The reactants were stirred at 333-343 $\mathrm{K}$ for $24 \mathrm{~h}$. After completion of the reaction, the reaction mass was extracted into diethyl ether $(2 \times 30 \mathrm{ml})$. The combined organic layers were dried over anhydrous sodium sulfate, concentrated and purified by

Table 1

Experimental details.

\begin{tabular}{ll}
\hline Crystal data & \\
Chemical formula & $\mathrm{C}_{19} \mathrm{H}_{22} \mathrm{~N}_{2} \mathrm{O}_{3}$ \\
$M_{\mathrm{r}}$ & 326.38 \\
Crystal system, space group & Monoclinic, $P 2_{1}$ \\
Temperature $(\mathrm{K})$ & 90 \\
$a, b, c(\AA)$ & $10.3526(2), 7.2612(1), 11.9198(2)$ \\
$\beta\left({ }^{\circ}\right.$ & $108.1210(6)$ \\
$V\left(\AA^{3}\right)$ & $851.60(2)$ \\
$Z$ & 2 \\
Radiation type & $\mathrm{Cu}$ K $\alpha$ \\
$\mu\left(\mathrm{mm}^{-1}\right)$ & 0.70 \\
Crystal size $(\mathrm{mm})$ & $0.25 \times 0.13 \times 0.10$ \\
& \\
Data collection & Bruker X8 Proteum \\
Diffractometer & Multi-scan $(S A D A B S ;$ Bruker, \\
Absorption correction & $2006)$ \\
& $0.850,0.942$ \\
$T_{\text {min }}, T_{\text {max }}$ & $11370,3020,3013$ \\
No. of measured, independent and & \\
observed $[I>2 \sigma(I)]$ reflections & 0.032 \\
$R_{\text {int }}(\text { sin } \theta / \lambda)_{\text {max }}\left(\AA^{-1}\right)$ & 0.602 \\
& \\
Refinement & \\
$R\left[F^{2}>2 \sigma\left(F^{2}\right)\right], w R\left(F^{2}\right), S$ & $0.027,0.068,1.05$ \\
No. of reflections & 3020 \\
No. of parameters & 220 \\
No. of restraints & 1 \\
H-atom treatment & H-atom parameters constrained \\
$\Delta \rho_{\text {max }}, \Delta \rho_{\text {min }}\left(\mathrm{e} \AA^{-3}\right)$ & $0.15,-0.14$ \\
Absolute structure & Flack $x$ determined using 1354 \\
& quotients $\left[\left(I^{+}\right)-\left(I^{-}\right)\right] /\left[\left(I^{+}\right)+\left(I^{-}\right)\right]$ \\
Absolute structure parameter & $-0.04(3)$ \\
\hline &
\end{tabular}

Computer programs: APEX2 and SAINT (Bruker, 2006), SHELXS97, SHELXTL and $X P$ in SHELXTL (Sheldrick, 2008), SHELXL2014 (Sheldrick, 2015) and CIFFIX (Parkin, 2013). 
column chromatography. The title compound was recrystallized from a mixture of hexane and acetone (9:1), which gave colourless needles upon slow evaporation of the solution at room temperature over $24 \mathrm{~h}$.

\section{Refinement}

Crystal data, data collection and structure refinement details are summarized in Table $1 . \mathrm{H}$ atoms were found in difference Fourier maps, but subsequently included in the refinement using riding models, with constrained distances set to $0.95 \AA$ $\left(\mathrm{Csp}{ }^{2} \mathrm{H}\right), 0.98 \AA\left(R \mathrm{CH}_{3}\right), 0.99 \AA\left(R_{2} \mathrm{CH}_{2}\right)$ and $1.00 \AA\left(R_{3} \mathrm{CH}\right)$. $U_{\text {iso }}(\mathrm{H})$ parameters were set to values of either $1.2 U_{\text {eq }}$ or $1.5 U_{\text {eq }}\left(R \mathrm{CH}_{3}\right.$ only) of the attached atom. The absolute structure parameter $[-0.04$ (3)] was determined directly from the diffraction data using 1354 Parsons quotients (Parsons et al., 2013), with the four chiral carbon atoms assigned to be $R, R, S, S$ for the arbitrarily numbered atoms $\mathrm{C} 4, \mathrm{C} 5, \mathrm{C} 6, \mathrm{C} 7$, respectively.

Refinement progress was checked using PLATON (Spek, 2009) and by an $R$-tensor (Parkin, 2000).

\section{Acknowledgements}

This work was supported by NIH/NCI grant CA158275.

\section{References}

Awang, D. V. C. (1989). Can. Pharm. J. 122, 266-270.

Bartsch, H.-H., Jarchow, O. \& Schmalle, H. W. (1983). Z. Kristallogr. 162, 15-17.
Bruker (2006). APEX2, SAINT and SADABS. Bruker-AXS Inc., Madison, Wisconsin, USA.

Castañeda-Acosta, J., Fischer, N. H. \& Vargas, D. (1993). J. Nat. Prod. 56, 90-98.

Gopal, Y. N. V., Arora, T. S. \& Van Dyke, M. W. (2007). Chem. Biol. 14, 813-823.

Groom, C. R. \& Allen, F. H. (2014). Angew. Chem. Int. Ed. 53, 662671.

Guzman, M. L., Rossi, R. M., Neelakantan, S., Li, X., Corbett, C. A., Hassane, D. C., Becker, M. W., Bennett, J. M., Sullivan, E., Lachowicz, J. L., Vaughan, A., Sweeney, C. J., Matthews, W., Carroll, M., Liesveld, J. L., Crooks, P. A. \& Jordan, C. T. (2007). Blood, 110, 4427-4435.

Han, C., Barrios, F. J., Riofski, M. V. \& Colby, D. A. (2009). J. Org. Chem. 74, 7176-7179.

Nasim, S. \& Crooks, P. A. (2008). Bioorg. Med. Chem. Lett. 18, 38703873.

Parkin, S. (2000). Acta Cryst. A56, 157-162.

Parkin, S. (2013). CIFFIX. http://xray.uky.edu/people/parkin/ programs/ciffix

Parsons, S., Flack, H. D. \& Wagner, T. (2013). Acta Cryst. B69, 249259.

Penthala, N. R., Bommagani, S., Janganati, V., MacNicol, K. B., Cragle, C. E., Madadi, N. R., Hardy, L. L., MacNicol, A. M. \& Crooks, P. A. (2014a). Eur. J. Med. Chem. 85, 517-525.

Penthala, N. R., Bommagani, S., Janganati, V., Parkin, S. \& Crooks, P. A. (2014b). Acta Cryst. E70, o1092-o1093.

Penthala, N. R., Janganati, V., Parkin, S., Varughese, K. I. \& Crooks, P. A. (2013). Acta Cryst. E69, o1709-o1710.

Picman, A. K. (1986). Biochem. Syst. Ecol. 14, 255-281.

Quick, A. \& Rogers, D. (1976). J. Chem. Soc. Perkin Trans. 2, pp. 465469.

Sheldrick, G. M. (2008). Acta Cryst. A64, 112-122.

Sheldrick, G. M. (2015). Acta Cryst. C71, 3-8.

Spek, A. L. (2009). Acta Cryst. D65, 148-155. 


\title{
supporting information
}

Acta Cryst. (2015). E71, 1536-1538 [doi:10.1107/S2056989015021507]

\section{Crystal structure of $(E)$-13-(pyrimidin-5-yl)parthenolide}

\author{
Shobanbabu Bommagani, Narsimha R. Penthala, Sean Parkin and Peter A. Crooks
}

Computing details

Data collection: APEX2 (Bruker, 2006); cell refinement: SAINT (Bruker, 2006); data reduction: SAINT (Bruker, 2006); program(s) used to solve structure: SHELXS97 (Sheldrick, 2008); program(s) used to refine structure: SHELXL2014 (Sheldrick, 2015); molecular graphics: XP in SHELXTL (Sheldrick, 2008); software used to prepare material for publication: SHELXTL (Sheldrick, 2008) and CIFFIX (Parkin, 2013).

(1aR,4E,7aS,8E,10aS,10bR)-1a,5-\ Dimethyl-8-[(pyrimidin-5-yl)methylidene]-2,3,6,7,7a,8,10a,10b-\ octahydrooxireno $\left[2^{\prime}, 3^{\prime}: 9,10\right]$ cyclodeca[1,2-b] furan-9(1aH)-one

Crystal data

$\mathrm{C}_{19} \mathrm{H}_{22} \mathrm{~N}_{2} \mathrm{O}_{3}$

$M_{r}=326.38$

Monoclinic, $P 2_{1}$

$a=10.3526(2) \AA$

$b=7.2612(1) \AA$

$c=11.9198(2) \AA$

$\beta=108.1210(6)^{\circ}$

$V=851.60(2) \AA^{3}$

$Z=2$

\section{Data collection}

\section{Bruker X8 Proteum} diffractometer

Radiation source: fine-focus rotating anode Detector resolution: 5.6 pixels $\mathrm{mm}^{-1}$

$\varphi$ and $\omega$ scans

Absorption correction: multi-scan

(SADABS; Bruker, 2006)

$T_{\min }=0.850, T_{\max }=0.942$

\section{Refinement}

Refinement on $F^{2}$

Least-squares matrix: full

$R\left[F^{2}>2 \sigma\left(F^{2}\right)\right]=0.027$

$w R\left(F^{2}\right)=0.068$

$S=1.05$

3020 reflections

220 parameters

1 restraint

Primary atom site location: structure-invariant direct methods
$F(000)=348$

$D_{\mathrm{x}}=1.273 \mathrm{Mg} \mathrm{m}^{-3}$

$\mathrm{Cu} K \alpha$ radiation, $\lambda=1.54178 \AA$

Cell parameters from 9908 reflections

$\theta=4.5-68.2^{\circ}$

$\mu=0.70 \mathrm{~mm}^{-1}$

$T=90 \mathrm{~K}$

Solvent-rounded block, colourless

$0.25 \times 0.13 \times 0.10 \mathrm{~mm}$

11370 measured reflections

3020 independent reflections

3013 reflections with $I>2 \sigma(I)$

$R_{\text {int }}=0.032$

$\theta_{\max }=68.2^{\circ}, \theta_{\min }=4.5^{\circ}$

$h=-12 \rightarrow 10$

$k=-8 \rightarrow 8$

$l=-14 \rightarrow 13$

Secondary atom site location: difference Fourier map

Hydrogen site location: difference Fourier map

$\mathrm{H}$-atom parameters constrained

$w=1 /\left[\sigma^{2}\left(F_{\mathrm{o}}^{2}\right)+(0.0326 P)^{2}+0.1773 P\right]$

where $P=\left(F_{\mathrm{o}}^{2}+2 F_{\mathrm{c}}^{2}\right) / 3$

$(\Delta / \sigma)_{\max }<0.001$

$\Delta \rho_{\max }=0.15$ e $\AA^{-3}$

$\Delta \rho_{\min }=-0.13$ e $\AA^{-3}$

Extinction correction: SHELXL-2014, $\mathrm{Fc}^{*}=\mathrm{kFc}\left[1+0.001 \mathrm{xFc}^{2} \lambda^{3} / \sin (2 \theta)\right]^{-1 / 4}$ 
Extinction coefficient: $0.010(2)$

\author{
Absolute structure: Flack x determined using \\ 1354 quotients $\left[\left(I^{+}\right)-\left(I^{-}\right)\right] /\left[\left(I^{+}\right)+\left(I^{-}\right)\right]$(Parsons et \\ al., 2013).
}

Absolute structure parameter: $-0.04(3)$

\section{Special details}

Experimental. The crystal was mounted with polyisobutene oil on the tip of a fine glass fibre, which was fastened in a copper mounting pin with electrical solder. It was placed directly into the cold gas stream of a liquid nitrogen based cryostat, according to published methods.

Diffraction data were collected with the crystal at $90 \mathrm{~K}$, which is standard practice in this laboratory for the majority of flash-cooled crystals.

The crystals were large, and could not be cut to size without inducing damage by crushing, leading to shattered, frayed ends. These damaged parts could easily be dissolved away, however, to give solvent-rounded undamaged pieces of optimal size for data collection.

Geometry. All e.s.d.'s (except the e.s.d. in the dihedral angle between two 1.s. planes) are estimated using the full covariance matrix. The cell e.s.d.'s are taken into account individually in the estimation of e.s.d.'s in distances, angles and torsion angles; correlations between e.s.d.'s in cell parameters are only used when they are defined by crystal symmetry. An approximate (isotropic) treatment of cell e.s.d.'s is used for estimating e.s.d.'s involving 1.s. planes.

Refinement. Refinement progress was checked using PLATON (Spek, 2009) and by an R-tensor (Parkin, 2000). The final model was further checked with the IUCr utility checkCIF.

Fractional atomic coordinates and isotropic or equivalent isotropic displacement parameters $\left(\AA^{2}\right)$

\begin{tabular}{|c|c|c|c|c|}
\hline & $x$ & $y$ & $z$ & $U_{\text {iso }} * / U_{\text {eq }}$ \\
\hline $\mathrm{O} 1$ & $0.59428(12)$ & 0.24072 (19) & $0.48957(10)$ & $0.0228(3)$ \\
\hline $\mathrm{O} 2$ & $0.30633(12)$ & $0.25486(17)$ & $0.43887(10)$ & $0.0195(3)$ \\
\hline $\mathrm{O} 3$ & $0.12397(13)$ & 0.29255 (19) & 0.49855 (11) & $0.0266(3)$ \\
\hline $\mathrm{C} 1$ & $0.57679(17)$ & $0.5285(2)$ & 0.19945 (14) & $0.0185(4)$ \\
\hline $\mathrm{H} 1 \mathrm{~A}$ & 0.5833 & 0.6439 & 0.2384 & $0.022 *$ \\
\hline $\mathrm{C} 2$ & $0.70386(17)$ & $0.4134(3)$ & $0.23289(16)$ & $0.0208(4)$ \\
\hline $\mathrm{H} 2 \mathrm{~A}$ & 0.7809 & 0.4881 & 0.2257 & $0.025^{*}$ \\
\hline $\mathrm{H} 2 \mathrm{~B}$ & 0.6915 & 0.3076 & 0.1781 & $0.025^{*}$ \\
\hline $\mathrm{C} 3$ & $0.73676(17)$ & $0.3417(3)$ & $0.36116(15)$ & $0.0202(4)$ \\
\hline $\mathrm{H} 3 \mathrm{~A}$ & 0.8146 & 0.2554 & 0.3792 & $0.024 *$ \\
\hline H3B & 0.7615 & 0.4460 & 0.4173 & $0.024^{*}$ \\
\hline $\mathrm{C} 4$ & $0.61322(17)$ & $0.2447(2)$ & $0.37345(14)$ & $0.0178(4)$ \\
\hline $\mathrm{C} 5$ & $0.51035(16)$ & $0.3615(2)$ & $0.40010(13)$ & $0.0165(3)$ \\
\hline $\mathrm{H} 5 \mathrm{~A}$ & 0.5353 & 0.4948 & 0.4118 & $0.020 *$ \\
\hline C6 & $0.35977(16)$ & $0.3252(2)$ & $0.34753(14)$ & $0.0159(3)$ \\
\hline H6A & 0.3445 & 0.2321 & 0.2829 & $0.019 *$ \\
\hline $\mathrm{C} 7$ & $0.27894(16)$ & $0.5036(2)$ & $0.29783(13)$ & $0.0149(3)$ \\
\hline $\mathrm{H} 7 \mathrm{~A}$ & 0.3396 & 0.6113 & 0.3292 & $0.018 *$ \\
\hline $\mathrm{C} 8$ & $0.22800(16)$ & 0.5184 & $0.16174(14)$ & $0.0176(4)$ \\
\hline H8A & 0.1434 & 0.5923 & 0.1376 & $0.021 *$ \\
\hline H8B & 0.2058 & 0.3936 & 0.1278 & $0.021 *$ \\
\hline C9 & $0.33322(17)$ & 0.6078 & $0.11062(14)$ & $0.0185(4)$ \\
\hline H9A & 0.2884 & 0.6358 & 0.0261 & $0.022^{*}$ \\
\hline H9B & 0.3638 & 0.7258 & 0.1519 & $0.022^{*}$ \\
\hline $\mathrm{C} 10$ & $0.45591(17)$ & $0.4887(2)$ & $0.12176(14)$ & $0.0179(4)$ \\
\hline C11 & $0.16862(16)$ & $0.5027(2)$ & $0.35605(14)$ & $0.0159(3)$ \\
\hline
\end{tabular}




$\begin{array}{lllll}\text { C12 } & 0.19100(17) & 0.3425(3) & 0.43718(14) & 0.0184(4) \\ \text { C13 } & 0.06867(17) & 0.6212(3) & 0.35248(14) & 0.0179(4) \\ \text { H13A } & 0.0096 & 0.5872 & 0.3961 & 0.021^{*} \\ \text { C14 } & 0.42876(19) & 0.3267(3) & 0.03864(16) & 0.0269(4) \\ \text { H14A } & 0.5140 & 0.2609 & 0.0473 & 0.040^{*} \\ \text { H14B } & 0.3908 & 0.3705 & -0.0428 & 0.040^{*} \\ \text { H14C } & 0.3639 & 0.2434 & 0.0573 & 0.040^{*} \\ \text { C15 } & 0.57578(18) & 0.0643(2) & 0.30934(18) & 0.0239(4) \\ \text { H15A } & 0.5012 & 0.0072 & 0.3311 & 0.036^{*} \\ \text { H15B } & 0.6548 & -0.0179 & 0.3312 & 0.036^{*} \\ \text { H15C } & 0.5470 & 0.0861 & 0.2240 & 0.036^{*} \\ \text { C16 } & 0.03901(16) & 0.7977(2) & 0.28928(14) & 0.0180(4) \\ \text { C17 } & 0.13568(18) & 0.9097(3) & 0.26601(18) & 0.0244(4) \\ \text { H17A } & 0.2281 & 0.8719 & 0.2933 & 0.029^{*} \\ \text { N18 } & 0.10538(16) & 1.0688(2) & 0.20684(16) & 0.0293(4) \\ \text { C19 } & -0.02533(19) & 1.1156(3) & 0.17269(17) & 0.0252(4) \\ \text { H19A } & -0.0485 & 1.2265 & 0.1287 & 0.030^{*} \\ \text { N20 } & -0.12697(15) & 1.0248(2) & 0.19312(14) & 0.0256(4) \\ \text { C21 } & -0.09291(18) & 0.8673(3) & 0.25279(15) & 0.0219(4) \\ \text { H21A } & -0.1621 & 0.7994 & 0.2713 & 0.026^{*}\end{array}$

Atomic displacement parameters $\left(\AA^{2}\right)$

\begin{tabular}{|c|c|c|c|c|c|c|}
\hline & $U^{11}$ & $U^{22}$ & $U^{33}$ & $U^{12}$ & $U^{13}$ & $U^{23}$ \\
\hline $\mathrm{O} 1$ & $0.0188(6)$ & $0.0306(7)$ & $0.0176(6)$ & $0.0061(5)$ & $0.0035(5)$ & $0.0092(5)$ \\
\hline $\mathrm{O} 2$ & $0.0187(6)$ & $0.0200(6)$ & $0.0221(6)$ & $0.0038(5)$ & $0.0098(5)$ & $0.0064(5)$ \\
\hline $\mathrm{O} 3$ & $0.0242(6)$ & $0.0305(8)$ & $0.0303(7)$ & $0.0031(6)$ & $0.0162(5)$ & 0.0097 (6) \\
\hline $\mathrm{C} 1$ & $0.0218(8)$ & $0.0163(8)$ & $0.0197(8)$ & $-0.0006(7)$ & $0.0100(7)$ & $0.0024(7)$ \\
\hline $\mathrm{C} 2$ & $0.0184(8)$ & $0.0208(9)$ & $0.0254(9)$ & $-0.0018(7)$ & $0.0101(7)$ & $-0.0001(7)$ \\
\hline $\mathrm{C} 3$ & $0.0157(8)$ & $0.0216(9)$ & $0.0228(8)$ & $0.0016(7)$ & $0.0053(6)$ & $-0.0004(7)$ \\
\hline $\mathrm{C} 4$ & $0.0163(8)$ & $0.0184(8)$ & $0.0174(8)$ & $0.0036(7)$ & $0.0031(6)$ & $0.0040(7)$ \\
\hline $\mathrm{C} 5$ & $0.0175(8)$ & $0.0173(8)$ & $0.0141(7)$ & $0.0020(7)$ & $0.0043(6)$ & $0.0031(6)$ \\
\hline C6 & $0.0173(8)$ & $0.0162(8)$ & $0.0154(7)$ & $0.0002(6)$ & $0.0068(6)$ & $0.0012(6)$ \\
\hline $\mathrm{C} 7$ & $0.0155(7)$ & $0.0147(8)$ & $0.0142(7)$ & $-0.0011(6)$ & $0.0041(6)$ & $-0.0008(6)$ \\
\hline $\mathrm{C} 8$ & $0.0172(8)$ & $0.0201(8)$ & $0.0143(8)$ & $0.0005(7)$ & $0.0034(6)$ & $0.0003(7)$ \\
\hline $\mathrm{C} 9$ & $0.0209(8)$ & $0.0195(8)$ & $0.0147(7)$ & $0.0018(7)$ & $0.0051(6)$ & $0.0044(7)$ \\
\hline $\mathrm{C} 10$ & $0.0221(8)$ & $0.0190(9)$ & $0.0150(7)$ & $0.0003(7)$ & $0.0092(7)$ & $0.0026(7)$ \\
\hline $\mathrm{C} 11$ & $0.0153(7)$ & $0.0170(8)$ & $0.0143(7)$ & $-0.0021(7)$ & $0.0031(6)$ & $-0.0010(7)$ \\
\hline $\mathrm{C} 12$ & $0.0166(8)$ & $0.0206(9)$ & $0.0187(8)$ & $0.0004(7)$ & $0.0065(6)$ & $0.0014(7)$ \\
\hline $\mathrm{C} 13$ & $0.0144(8)$ & $0.0213(9)$ & $0.0175(8)$ & $-0.0011(7)$ & $0.0044(6)$ & $-0.0018(7)$ \\
\hline $\mathrm{C} 14$ & $0.0258(9)$ & $0.0321(11)$ & $0.0219(8)$ & $0.0041(8)$ & $0.0061(7)$ & $-0.0061(8)$ \\
\hline $\mathrm{C} 15$ & $0.0206(8)$ & $0.0177(9)$ & $0.0346(10)$ & $0.0023(7)$ & $0.0105(8)$ & $0.0005(7)$ \\
\hline $\mathrm{C} 16$ & $0.0175(8)$ & $0.0190(9)$ & $0.0161(7)$ & 0.0009 (7) & $0.0032(6)$ & $-0.0044(7)$ \\
\hline $\mathrm{C} 17$ & $0.0156(8)$ & $0.0189(9)$ & $0.0350(10)$ & $0.0005(7)$ & $0.0024(8)$ & $0.0019(8)$ \\
\hline N18 & $0.0209(8)$ & $0.0216(9)$ & $0.0409(10)$ & $-0.0014(6)$ & $0.0030(7)$ & $0.0062(7)$ \\
\hline $\mathrm{C} 19$ & $0.0240(9)$ & $0.0183(9)$ & $0.0288(9)$ & $0.0021(8)$ & $0.0016(7)$ & $0.0019(8)$ \\
\hline $\mathrm{N} 20$ & $0.0206(8)$ & $0.0232(8)$ & $0.0304(8)$ & $0.0042(6)$ & $0.0043(6)$ & $0.0003(7)$ \\
\hline $\mathrm{C} 21$ & $0.0194(8)$ & $0.0219(9)$ & $0.0253(9)$ & $0.0026(7)$ & $0.0084(7)$ & $-0.0024(7)$ \\
\hline
\end{tabular}


Geometric parameters $\left(\AA,{ }^{\circ}\right)$

\begin{tabular}{|c|c|c|c|}
\hline $\mathrm{O} 1-\mathrm{C} 5$ & $1.444(2)$ & $\mathrm{C} 8-\mathrm{H} 8 \mathrm{~B}$ & 0.9900 \\
\hline $\mathrm{O} 1-\mathrm{C} 4$ & $1.457(2)$ & $\mathrm{C} 9-\mathrm{C} 10$ & $1.508(2)$ \\
\hline $\mathrm{O} 2-\mathrm{C} 12$ & $1.348(2)$ & C9-H9A & 0.9900 \\
\hline $\mathrm{O} 2-\mathrm{C} 6$ & $1.4585(19)$ & C9-H9B & 0.9900 \\
\hline $\mathrm{O} 3-\mathrm{C} 12$ & $1.210(2)$ & $\mathrm{C} 10-\mathrm{C} 14$ & $1.507(3)$ \\
\hline $\mathrm{C} 1-\mathrm{C} 10$ & $1.337(2)$ & $\mathrm{C} 11-\mathrm{C} 13$ & $1.336(2)$ \\
\hline $\mathrm{C} 1-\mathrm{C} 2$ & $1.504(2)$ & $\mathrm{C} 11-\mathrm{C} 12$ & $1.484(2)$ \\
\hline $\mathrm{C} 1-\mathrm{H} 1 \mathrm{~A}$ & 0.9500 & $\mathrm{C} 13-\mathrm{C} 16$ & $1.470(2)$ \\
\hline $\mathrm{C} 2-\mathrm{C} 3$ & $1.549(2)$ & $\mathrm{C} 13-\mathrm{H} 13 \mathrm{~A}$ & 0.9500 \\
\hline $\mathrm{C} 2-\mathrm{H} 2 \mathrm{~A}$ & 0.9900 & $\mathrm{C} 14-\mathrm{H} 14 \mathrm{~A}$ & 0.9800 \\
\hline $\mathrm{C} 2-\mathrm{H} 2 \mathrm{~B}$ & 0.9900 & $\mathrm{C} 14-\mathrm{H} 14 \mathrm{~B}$ & 0.9800 \\
\hline $\mathrm{C} 3-\mathrm{C} 4$ & $1.507(2)$ & $\mathrm{C} 14-\mathrm{H} 14 \mathrm{C}$ & 0.9800 \\
\hline $\mathrm{C} 3-\mathrm{H} 3 \mathrm{~A}$ & 0.9900 & C15-H15A & 0.9800 \\
\hline C $3-\mathrm{H} 3 \mathrm{~B}$ & 0.9900 & C15-H15B & 0.9800 \\
\hline $\mathrm{C} 4-\mathrm{C} 5$ & $1.471(2)$ & $\mathrm{C} 15-\mathrm{H} 15 \mathrm{C}$ & 0.9800 \\
\hline $\mathrm{C} 4-\mathrm{C} 15$ & $1.505(3)$ & $\mathrm{C} 16-\mathrm{C} 17$ & $1.383(3)$ \\
\hline $\mathrm{C} 5-\mathrm{C} 6$ & $1.511(2)$ & $\mathrm{C} 16-\mathrm{C} 21$ & $1.393(2)$ \\
\hline $\mathrm{C} 5-\mathrm{H} 5 \mathrm{~A}$ & 1.0000 & C17-N18 & $1.339(2)$ \\
\hline $\mathrm{C} 6-\mathrm{C} 7$ & $1.556(2)$ & C17-H17A & 0.9500 \\
\hline C6-H6A & 1.0000 & N18-C19 & $1.330(2)$ \\
\hline $\mathrm{C} 7-\mathrm{C} 11$ & $1.510(2)$ & $\mathrm{C} 19-\mathrm{N} 20$ & $1.327(3)$ \\
\hline $\mathrm{C} 7-\mathrm{C} 8$ & $1.546(2)$ & C19-H19A & 0.9500 \\
\hline C7-H7A & 1.0000 & $\mathrm{~N} 20-\mathrm{C} 21$ & $1.335(3)$ \\
\hline $\mathrm{C} 8-\mathrm{C} 9$ & $1.547(2)$ & $\mathrm{C} 21-\mathrm{H} 21 \mathrm{~A}$ & 0.9500 \\
\hline $\mathrm{C} 8-\mathrm{H} 8 \mathrm{~A}$ & 0.9900 & & \\
\hline $\mathrm{C} 5-\mathrm{O} 1-\mathrm{C} 4$ & $60.94(10)$ & $\mathrm{H} 8 \mathrm{~A}-\mathrm{C} 8-\mathrm{H} 8 \mathrm{~B}$ & 107.8 \\
\hline $\mathrm{C} 12-\mathrm{O} 2-\mathrm{C} 6$ & $111.31(12)$ & $\mathrm{C} 10-\mathrm{C} 9-\mathrm{C} 8$ & $113.70(14)$ \\
\hline $\mathrm{C} 10-\mathrm{C} 1-\mathrm{C} 2$ & $128.05(17)$ & $\mathrm{C} 10-\mathrm{C} 9-\mathrm{H} 9 \mathrm{~A}$ & 108.8 \\
\hline $\mathrm{C} 10-\mathrm{C} 1-\mathrm{H} 1 \mathrm{~A}$ & 116.0 & $\mathrm{C} 8-\mathrm{C} 9-\mathrm{H} 9 \mathrm{~A}$ & 108.8 \\
\hline $\mathrm{C} 2-\mathrm{C} 1-\mathrm{H} 1 \mathrm{~A}$ & 116.0 & $\mathrm{C} 10-\mathrm{C} 9-\mathrm{H} 9 \mathrm{~B}$ & 108.8 \\
\hline $\mathrm{C} 1-\mathrm{C} 2-\mathrm{C} 3$ & $110.73(14)$ & $\mathrm{C} 8-\mathrm{C} 9-\mathrm{H} 9 \mathrm{~B}$ & 108.8 \\
\hline $\mathrm{C} 1-\mathrm{C} 2-\mathrm{H} 2 \mathrm{~A}$ & 109.5 & $\mathrm{H} 9 \mathrm{~A}-\mathrm{C} 9-\mathrm{H} 9 \mathrm{~B}$ & 107.7 \\
\hline $\mathrm{C} 3-\mathrm{C} 2-\mathrm{H} 2 \mathrm{~A}$ & 109.5 & $\mathrm{C} 1-\mathrm{C} 10-\mathrm{C} 14$ & $124.58(16)$ \\
\hline $\mathrm{C} 1-\mathrm{C} 2-\mathrm{H} 2 \mathrm{~B}$ & 109.5 & $\mathrm{C} 1-\mathrm{C} 10-\mathrm{C} 9$ & $121.22(16)$ \\
\hline $\mathrm{C} 3-\mathrm{C} 2-\mathrm{H} 2 \mathrm{~B}$ & 109.5 & $\mathrm{C} 14-\mathrm{C} 10-\mathrm{C} 9$ & $114.20(15)$ \\
\hline $\mathrm{H} 2 \mathrm{~A}-\mathrm{C} 2-\mathrm{H} 2 \mathrm{~B}$ & 108.1 & $\mathrm{C} 13-\mathrm{C} 11-\mathrm{C} 12$ & $119.20(15)$ \\
\hline $\mathrm{C} 4-\mathrm{C} 3-\mathrm{C} 2$ & $108.68(14)$ & $\mathrm{C} 13-\mathrm{C} 11-\mathrm{C} 7$ & $132.19(16)$ \\
\hline $\mathrm{C} 4-\mathrm{C} 3-\mathrm{H} 3 \mathrm{~A}$ & 110.0 & $\mathrm{C} 12-\mathrm{C} 11-\mathrm{C} 7$ & $108.42(13)$ \\
\hline $\mathrm{C} 2-\mathrm{C} 3-\mathrm{H} 3 \mathrm{~A}$ & 110.0 & $\mathrm{O} 3-\mathrm{C} 12-\mathrm{O} 2$ & $121.43(16)$ \\
\hline $\mathrm{C} 4-\mathrm{C} 3-\mathrm{H} 3 \mathrm{~B}$ & 110.0 & $\mathrm{O} 3-\mathrm{C} 12-\mathrm{C} 11$ & $128.78(16)$ \\
\hline $\mathrm{C} 2-\mathrm{C} 3-\mathrm{H} 3 \mathrm{~B}$ & 110.0 & $\mathrm{O} 2-\mathrm{C} 12-\mathrm{C} 11$ & $109.75(13)$ \\
\hline $\mathrm{H} 3 \mathrm{~A}-\mathrm{C} 3-\mathrm{H} 3 \mathrm{~B}$ & 108.3 & $\mathrm{C} 11-\mathrm{C} 13-\mathrm{C} 16$ & $127.89(16)$ \\
\hline $\mathrm{O} 1-\mathrm{C} 4-\mathrm{C} 5$ & $59.08(10)$ & $\mathrm{C} 11-\mathrm{C} 13-\mathrm{H} 13 \mathrm{~A}$ & 116.1 \\
\hline $\mathrm{O} 1-\mathrm{C} 4-\mathrm{C} 15$ & $112.05(15)$ & $\mathrm{C} 16-\mathrm{C} 13-\mathrm{H} 13 \mathrm{~A}$ & 116.1 \\
\hline $\mathrm{C} 5-\mathrm{C} 4-\mathrm{C} 15$ & $121.46(15)$ & $\mathrm{C} 10-\mathrm{C} 14-\mathrm{H} 14 \mathrm{~A}$ & 109.5 \\
\hline
\end{tabular}




\begin{tabular}{|c|c|c|c|}
\hline $\mathrm{O} 1-\mathrm{C} 4-\mathrm{C} 3$ & $118.08(14)$ & $\mathrm{C} 10-\mathrm{C} 14-\mathrm{H} 14 \mathrm{~B}$ & 109.5 \\
\hline $\mathrm{C} 5-\mathrm{C} 4-\mathrm{C} 3$ & $116.40(15)$ & $\mathrm{H} 14 \mathrm{~A}-\mathrm{C} 14-\mathrm{H} 14 \mathrm{~B}$ & 109.5 \\
\hline $\mathrm{C} 15-\mathrm{C} 4-\mathrm{C} 3$ & $116.73(15)$ & $\mathrm{C} 10-\mathrm{C} 14-\mathrm{H} 14 \mathrm{C}$ & 109.5 \\
\hline $\mathrm{O} 1-\mathrm{C} 5-\mathrm{C} 4$ & $59.97(10)$ & $\mathrm{H} 14 \mathrm{~A}-\mathrm{C} 14-\mathrm{H} 14 \mathrm{C}$ & 109.5 \\
\hline $\mathrm{O} 1-\mathrm{C} 5-\mathrm{C} 6$ & $120.41(14)$ & $\mathrm{H} 14 \mathrm{~B}-\mathrm{C} 14-\mathrm{H} 14 \mathrm{C}$ & 109.5 \\
\hline $\mathrm{C} 4-\mathrm{C} 5-\mathrm{C} 6$ & $122.16(14)$ & $\mathrm{C} 4-\mathrm{C} 15-\mathrm{H} 15 \mathrm{~A}$ & 109.5 \\
\hline $\mathrm{O} 1-\mathrm{C} 5-\mathrm{H} 5 \mathrm{~A}$ & 114.5 & $\mathrm{C} 4-\mathrm{C} 15-\mathrm{H} 15 \mathrm{~B}$ & 109.5 \\
\hline $\mathrm{C} 4-\mathrm{C} 5-\mathrm{H} 5 \mathrm{~A}$ & 114.5 & $\mathrm{H} 15 \mathrm{~A}-\mathrm{C} 15-\mathrm{H} 15 \mathrm{~B}$ & 109.5 \\
\hline $\mathrm{C} 6-\mathrm{C} 5-\mathrm{H} 5 \mathrm{~A}$ & 114.5 & $\mathrm{C} 4-\mathrm{C} 15-\mathrm{H} 15 \mathrm{C}$ & 109.5 \\
\hline $\mathrm{O} 2-\mathrm{C} 6-\mathrm{C} 5$ & $109.48(12)$ & $\mathrm{H} 15 \mathrm{~A}-\mathrm{C} 15-\mathrm{H} 15 \mathrm{C}$ & 109.5 \\
\hline $\mathrm{O} 2-\mathrm{C} 6-\mathrm{C} 7$ & $106.97(12)$ & $\mathrm{H} 15 \mathrm{~B}-\mathrm{C} 15-\mathrm{H} 15 \mathrm{C}$ & 109.5 \\
\hline $\mathrm{C} 5-\mathrm{C} 6-\mathrm{C} 7$ & $112.07(13)$ & $\mathrm{C} 17-\mathrm{C} 16-\mathrm{C} 21$ & $115.11(16)$ \\
\hline $\mathrm{O} 2-\mathrm{C} 6-\mathrm{H} 6 \mathrm{~A}$ & 109.4 & $\mathrm{C} 17-\mathrm{C} 16-\mathrm{C} 13$ & $124.52(15)$ \\
\hline $\mathrm{C} 5-\mathrm{C} 6-\mathrm{H} 6 \mathrm{~A}$ & 109.4 & $\mathrm{C} 21-\mathrm{C} 16-\mathrm{C} 13$ & $120.31(15)$ \\
\hline $\mathrm{C} 7-\mathrm{C} 6-\mathrm{H} 6 \mathrm{~A}$ & 109.4 & $\mathrm{~N} 18-\mathrm{C} 17-\mathrm{C} 16$ & $123.19(16)$ \\
\hline $\mathrm{C} 11-\mathrm{C} 7-\mathrm{C} 8$ & $115.01(13)$ & N18-C17-H17A & 118.4 \\
\hline $\mathrm{C} 11-\mathrm{C} 7-\mathrm{C} 6$ & $102.45(13)$ & $\mathrm{C} 16-\mathrm{C} 17-\mathrm{H} 17 \mathrm{~A}$ & 118.4 \\
\hline $\mathrm{C} 8-\mathrm{C} 7-\mathrm{C} 6$ & $115.15(13)$ & $\mathrm{C} 19-\mathrm{N} 18-\mathrm{C} 17$ & $115.52(17)$ \\
\hline $\mathrm{C} 11-\mathrm{C} 7-\mathrm{H} 7 \mathrm{~A}$ & 107.9 & $\mathrm{~N} 20-\mathrm{C} 19-\mathrm{N} 18$ & $127.34(18)$ \\
\hline $\mathrm{C} 8-\mathrm{C} 7-\mathrm{H} 7 \mathrm{~A}$ & 107.9 & $\mathrm{~N} 20-\mathrm{C} 19-\mathrm{H} 19 \mathrm{~A}$ & 116.3 \\
\hline $\mathrm{C} 6-\mathrm{C} 7-\mathrm{H} 7 \mathrm{~A}$ & 107.9 & N18-C19-H19A & 116.3 \\
\hline $\mathrm{C} 7-\mathrm{C} 8-\mathrm{C} 9$ & $112.99(13)$ & $\mathrm{C} 19-\mathrm{N} 20-\mathrm{C} 21$ & $115.48(15)$ \\
\hline $\mathrm{C} 7-\mathrm{C} 8-\mathrm{H} 8 \mathrm{~A}$ & 109.0 & $\mathrm{~N} 20-\mathrm{C} 21-\mathrm{C} 16$ & $123.23(17)$ \\
\hline $\mathrm{C} 9-\mathrm{C} 8-\mathrm{H} 8 \mathrm{~A}$ & 109.0 & $\mathrm{~N} 20-\mathrm{C} 21-\mathrm{H} 21 \mathrm{~A}$ & 118.4 \\
\hline $\mathrm{C} 7-\mathrm{C} 8-\mathrm{H} 8 \mathrm{~B}$ & 109.0 & $\mathrm{C} 16-\mathrm{C} 21-\mathrm{H} 21 \mathrm{~A}$ & 118.4 \\
\hline $\mathrm{C} 9-\mathrm{C} 8-\mathrm{H} 8 \mathrm{~B}$ & 109.0 & & \\
\hline $\mathrm{C} 10-\mathrm{C} 1-\mathrm{C} 2-\mathrm{C} 3$ & $-110.99(19)$ & $\mathrm{C} 2-\mathrm{C} 1-\mathrm{C} 10-\mathrm{C} 14$ & $-8.9(3)$ \\
\hline $\mathrm{C} 1-\mathrm{C} 2-\mathrm{C} 3-\mathrm{C} 4$ & $53.54(19)$ & $\mathrm{C} 2-\mathrm{C} 1-\mathrm{C} 10-\mathrm{C} 9$ & $171.47(16)$ \\
\hline $\mathrm{C} 5-\mathrm{O} 1-\mathrm{C} 4-\mathrm{C} 15$ & $-114.44(16)$ & $\mathrm{C} 8-\mathrm{C} 9-\mathrm{C} 10-\mathrm{C} 1$ & $-107.23(18)$ \\
\hline $\mathrm{C} 5-\mathrm{O} 1-\mathrm{C} 4-\mathrm{C} 3$ & $105.54(17)$ & $\mathrm{C} 8-\mathrm{C} 9-\mathrm{C} 10-\mathrm{C} 14$ & $73.07(19)$ \\
\hline $\mathrm{C} 2-\mathrm{C} 3-\mathrm{C} 4-\mathrm{O} 1$ & $-152.28(15)$ & $\mathrm{C} 8-\mathrm{C} 7-\mathrm{C} 11-\mathrm{C} 13$ & $-56.5(2)$ \\
\hline $\mathrm{C} 2-\mathrm{C} 3-\mathrm{C} 4-\mathrm{C} 5$ & $-84.95(18)$ & $\mathrm{C} 6-\mathrm{C} 7-\mathrm{C} 11-\mathrm{C} 13$ & $177.78(17)$ \\
\hline $\mathrm{C} 2-\mathrm{C} 3-\mathrm{C} 4-\mathrm{C} 15$ & $69.54(18)$ & $\mathrm{C} 8-\mathrm{C} 7-\mathrm{C} 11-\mathrm{C} 12$ & $128.73(15)$ \\
\hline $\mathrm{C} 4-\mathrm{O} 1-\mathrm{C} 5-\mathrm{C} 6$ & $111.94(17)$ & $\mathrm{C} 6-\mathrm{C} 7-\mathrm{C} 11-\mathrm{C} 12$ & $3.06(16)$ \\
\hline $\mathrm{C} 15-\mathrm{C} 4-\mathrm{C} 5-\mathrm{O} 1$ & $98.43(17)$ & $\mathrm{C} 6-\mathrm{O} 2-\mathrm{C} 12-\mathrm{O} 3$ & $172.90(16)$ \\
\hline $\mathrm{C} 3-\mathrm{C} 4-\mathrm{C} 5-\mathrm{O} 1$ & $-108.37(16)$ & $\mathrm{C} 6-\mathrm{O} 2-\mathrm{C} 12-\mathrm{C} 11$ & $-9.33(18)$ \\
\hline $\mathrm{O} 1-\mathrm{C} 4-\mathrm{C} 5-\mathrm{C} 6$ & $-109.10(17)$ & $\mathrm{C} 13-\mathrm{C} 11-\mathrm{C} 12-\mathrm{O} 3$ & $5.6(3)$ \\
\hline $\mathrm{C} 15-\mathrm{C} 4-\mathrm{C} 5-\mathrm{C} 6$ & $-10.7(2)$ & $\mathrm{C} 7-\mathrm{C} 11-\mathrm{C} 12-\mathrm{O} 3$ & $-178.86(18)$ \\
\hline $\mathrm{C} 3-\mathrm{C} 4-\mathrm{C} 5-\mathrm{C} 6$ & $142.53(15)$ & $\mathrm{C} 13-\mathrm{C} 11-\mathrm{C} 12-\mathrm{O} 2$ & $-171.94(15)$ \\
\hline $\mathrm{C} 12-\mathrm{O} 2-\mathrm{C} 6-\mathrm{C} 5$ & $132.85(14)$ & $\mathrm{C} 7-\mathrm{C} 11-\mathrm{C} 12-\mathrm{O} 2$ & $3.59(18)$ \\
\hline $\mathrm{C} 12-\mathrm{O} 2-\mathrm{C} 6-\mathrm{C} 7$ & $11.21(17)$ & $\mathrm{C} 12-\mathrm{C} 11-\mathrm{C} 13-\mathrm{C} 16$ & $172.57(15)$ \\
\hline $\mathrm{O} 1-\mathrm{C} 5-\mathrm{C} 6-\mathrm{O} 2$ & $36.7(2)$ & $\mathrm{C} 7-\mathrm{C} 11-\mathrm{C} 13-\mathrm{C} 16$ & $-1.7(3)$ \\
\hline $\mathrm{C} 4-\mathrm{C} 5-\mathrm{C} 6-\mathrm{O} 2$ & $108.24(17)$ & $\mathrm{C} 11-\mathrm{C} 13-\mathrm{C} 16-\mathrm{C} 17$ & $-29.8(3)$ \\
\hline $\mathrm{O} 1-\mathrm{C} 5-\mathrm{C} 6-\mathrm{C} 7$ & $155.20(14)$ & $\mathrm{C} 11-\mathrm{C} 13-\mathrm{C} 16-\mathrm{C} 21$ & $153.17(17)$ \\
\hline $\mathrm{C} 4-\mathrm{C} 5-\mathrm{C} 6-\mathrm{C} 7$ & $-133.25(15)$ & $\mathrm{C} 21-\mathrm{C} 16-\mathrm{C} 17-\mathrm{N} 18$ & $-3.7(3)$ \\
\hline $\mathrm{O} 2-\mathrm{C} 6-\mathrm{C} 7-\mathrm{C} 11$ & $-8.19(16)$ & $\mathrm{C} 13-\mathrm{C} 16-\mathrm{C} 17-\mathrm{N} 18$ & $179.10(17)$ \\
\hline $\mathrm{C} 5-\mathrm{C} 6-\mathrm{C} 7-\mathrm{C} 11$ & $-128.18(14)$ & $\mathrm{C} 16-\mathrm{C} 17-\mathrm{N} 18-\mathrm{C} 19$ & $1.1(3)$ \\
\hline
\end{tabular}




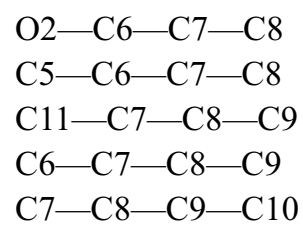

$\mathrm{O} 2-\mathrm{C} 6-\mathrm{C} 7-\mathrm{C} 8$

$\mathrm{C} 11-\mathrm{C} 7-\mathrm{C} 8-\mathrm{C} 9$

$\mathrm{C} 7-\mathrm{C} 8-\mathrm{C} 9-\mathrm{C} 10$
$-133.78(14)$

$106.23(15)$

$153.12(15)$

$-88.08(18)$

69.71 (19)

$\begin{array}{ll}\mathrm{C} 17-\mathrm{N} 18-\mathrm{C} 19-\mathrm{N} 20 & 1.7(3) \\ \mathrm{N} 18-\mathrm{C} 19-\mathrm{N} 20-\mathrm{C} 21 & -1.4(3) \\ \mathrm{C} 19-\mathrm{N} 20-\mathrm{C} 21-\mathrm{C} 16 & -1.7(3) \\ \mathrm{C} 17-\mathrm{C} 16-\mathrm{C} 21-\mathrm{N} 20 & 4.0(2) \\ \mathrm{C} 13-\mathrm{C} 16-\mathrm{C} 21-\mathrm{N} 20 & -178.66(16)\end{array}$

